'Facultad de Odontología, Universidad Finis Terrae, Santiago, Chile. 2Escuela de Enfermería, Facultad de Medicina, Universidad Finis Terrae, Red Salud CAPREDENA, Santiago, Chile.

${ }^{3}$ Escuela de Odontología, Facultad de Ciencias, Universidad Mayor, Santiago, Chile. ${ }^{4}$ Universidad Finis Terrae, Santiago, Chile. ${ }^{a}$ Cirujano Dentista.

${ }^{\text {b}}$ Enfermera Matrona. 'Alumnas Magíster en Docencia en Ciencias de la Salud, Universidad Finis Terrae. dPhd.

Recibido el 24 de octubre de 2016, aceptado el 23 de marzo de 2017.

Correspondencia a: Elisa Parraguez López Universidad Finis Terrae. Santiago, Chile. elisa.parraguez@gmail.com

\section{Efectividad de la simulación en la educación médica desde la perspectiva de seguridad de pacientes}

\author{
PATRICIA MOYA R., ${ }^{1, a}$, MAXY RUZ A.,, , \\ ELISA PARRAGUEZ L. .,a,c, VERÓNICA CARREÑO E.,.$^{3, a, c}$, \\ ANA MARÍA RODRÍGUEZ C. ${ }^{3, \mathrm{a}, \mathrm{c}}$, PATRICIA FROES M. ${ }^{4, \mathrm{~d}}$
}

\section{Simulation in medical education from the perspective of patients' safety}

We herein review the association between patients' safety and simulation methods for medical education. This evidence should help to change the present paradigm in medical education, where there is still reticence towards this education method. A total of 20 papers on the subject were reviewed. Ninety percent of these articles conclude that simulation contributes to patient safety, 5\% conclude that the evidence is uncertain and 5\% conclude that the effects will be seen in the next decade. Thus, the majority of papers support the use of simulation in medical education as a method that improves patients' safety.

(Rev Med Chile 2017; 145: 514-526)

Key words: Education; Education, Medical; Safety; Simulation Training.
U n elemento fundamental en la práctica médica es "Primum non nocere" sobre todo, no hacer daño. Sin embargo, la evidencia refiere que $10 \%$ de los pacientes ingresados en el hospital sufren de algún tipo de evento adverso o daño ${ }^{1}$.

Las investigaciones sobre eventos adversos (EA) han puesto de manifiesto la necesidad de mejorar la seguridad del paciente durante la atención sanitaria. Los eventos adversos son lesiones o complicaciones resultantes en la muerte, la discapacidad o la estancia hospitalaria prolongada que se derivan de la gestión de la atención de salud no deseados ${ }^{2}$.

El concepto de simulación hace referencia a representar algo, fingiendo o imitando lo que no es. Simular en el área de la salud, consiste en situar a un estudiante en un contexto que imite algún aspecto de la realidad clínica. Las simulaciones son técnicas educativas que se utilizan en el contexto de la denominada educación médica basada en las simulaciones que, en sentido amplio se define como cualquier actividad docente que utilice la ayuda de simuladores con el fin de estimular y favorecer el aprendizaje representando en lo posible un escenario clínico relativamente complejo ${ }^{3}$.

Según Ziv $(2009,2008)$ citado por Argullós et $\mathrm{al}^{3}$, se distinguen distintas categorías en el contexto de la simulación partiendo por los simuladores de uso específico y de baja tecnología, donde se replica sólo una parte del organismo y del ambiente permitiendo el desarrollo de habilidades psicomotoras básicas. Después se encuentran los simuladores virtuales en pantalla que permiten simular diversas situaciones e interactuar con los estudiantes, siendo su objetivo entrenar y evaluar conocimientos y toma de decisiones. A continuación los simuladores de tareas complejas logran una representación tridimensional de un espacio anatómico y permiten desarrollar habilidades manuales y de orientación tridimensional, adquirir conocimientos teóricos y mejorar la toma de decisiones. Y por último los simuladores de paciente completo, que son maniquíes de tamaño real y de 
manejo computacional que permiten el desarrollo de competencias en el manejo de situaciones clínicas complejas y en el trabajo en equipo ${ }^{3}$.

La educación médica basada en simulación ha demostrado ser eficaz en la trasferencia de conocimientos y habilidades que deben adquirir los profesionales de la salud de una manera segura y orientada hacia la educación ${ }^{1,4}$. En Chile, a pesar de que muchos grupos la han utilizado desde hace años, su inserción formal en los currículos de las Facultades de Medicina recién comienza. Existe reticencia por el uso de esta metodología de enseñanza-aprendizaje en el contexto de los aportes para la seguridad del paciente.

Este estudio busca realizar una revisión de la evidencia de experiencias extranjeras respecto a la educación médica basada en la metodología de simulación y su asociación con la seguridad del paciente, considerando utilizarla como evidencia en el cambio del paradigma actual existente en la educación médica. Se entiende por educación médica a la realizada en estudiantes universitarios de las carreras de la salud, en pre y post grado.

\section{Material y Método}

Para verificar el estado actual de las investigaciones y las contribuciones disponibles, se llevó a cabo una revisión de la literatura como estrategia metodológica. Para apoyar el proceso de planificación se formuló una pregunta de investigación. Se definieron diferentes referencias académicas de investigación presentes en Pubmed y Scielo. En esta etapa, se utilizaron términos consolidados de búsqueda formulados en inglés, con tres palabras claves: simulation, education, safety, encontrando 1.007 artículos relacionados.

El protocolo construido definió los siguientes criterios para la inclusión de los estudios: artículos publicados presentados en texto completo y de libre acceso (137), artículos publicados en los cinco años previos a la revisión (87) y relacionados con seres humanos que incluían pacientes y/o procedimientos simulados (67). Luego se hizo una revisión más exhaustiva, la cual consistió en una lectura de cada uno de los artículos, momento en el cual se eliminaron algunos por no estar relacionados directamente con el tema clínico, aquellos en otro idioma diferente al inglés o aquellos poco explícitos resultando un total de 20 (Figura 1).
Una vez aplicados estos criterios se dio por finalizada la etapa de planificación, lo que permitió la realización de la revisión, extracción de datos y construcción de una tabla.

\section{Resultados}

Para sustentar mejor el análisis y discusión de esta investigación, fueron seleccionados 20 artículos de un total de 1.007 encontrados en las bases de datos. Utilizando los métodos y criterios de inclusión citados, fue posible construir la Tabla 1.

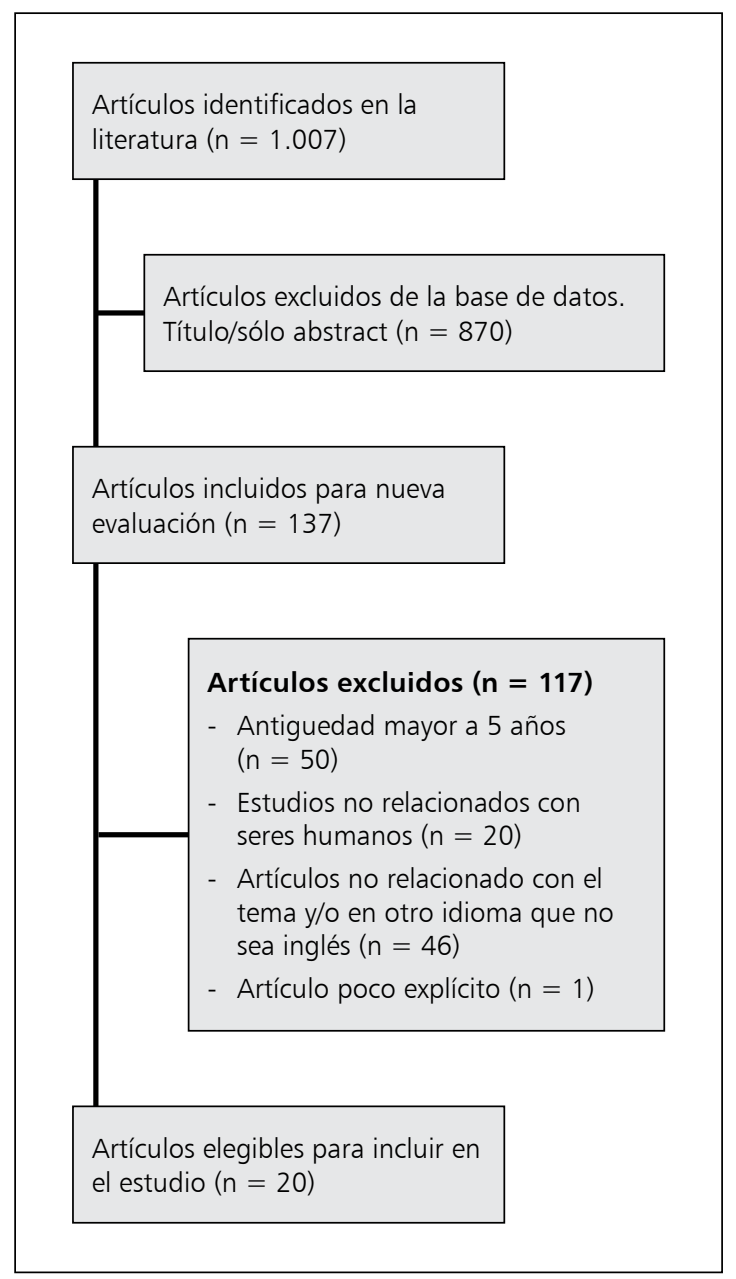

Figura 1. Diagrama de flujo del proceso de selección de los estudios. 


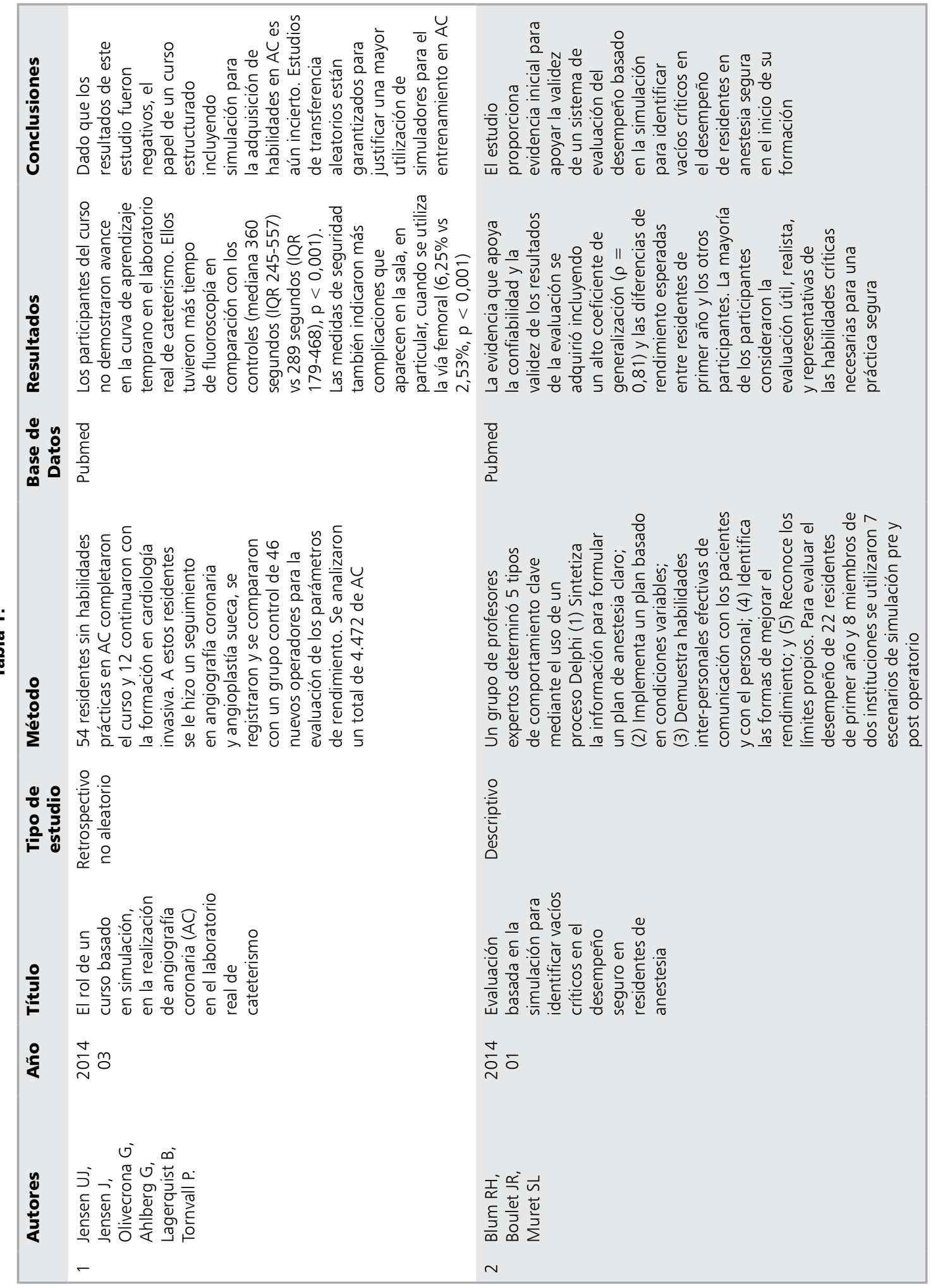




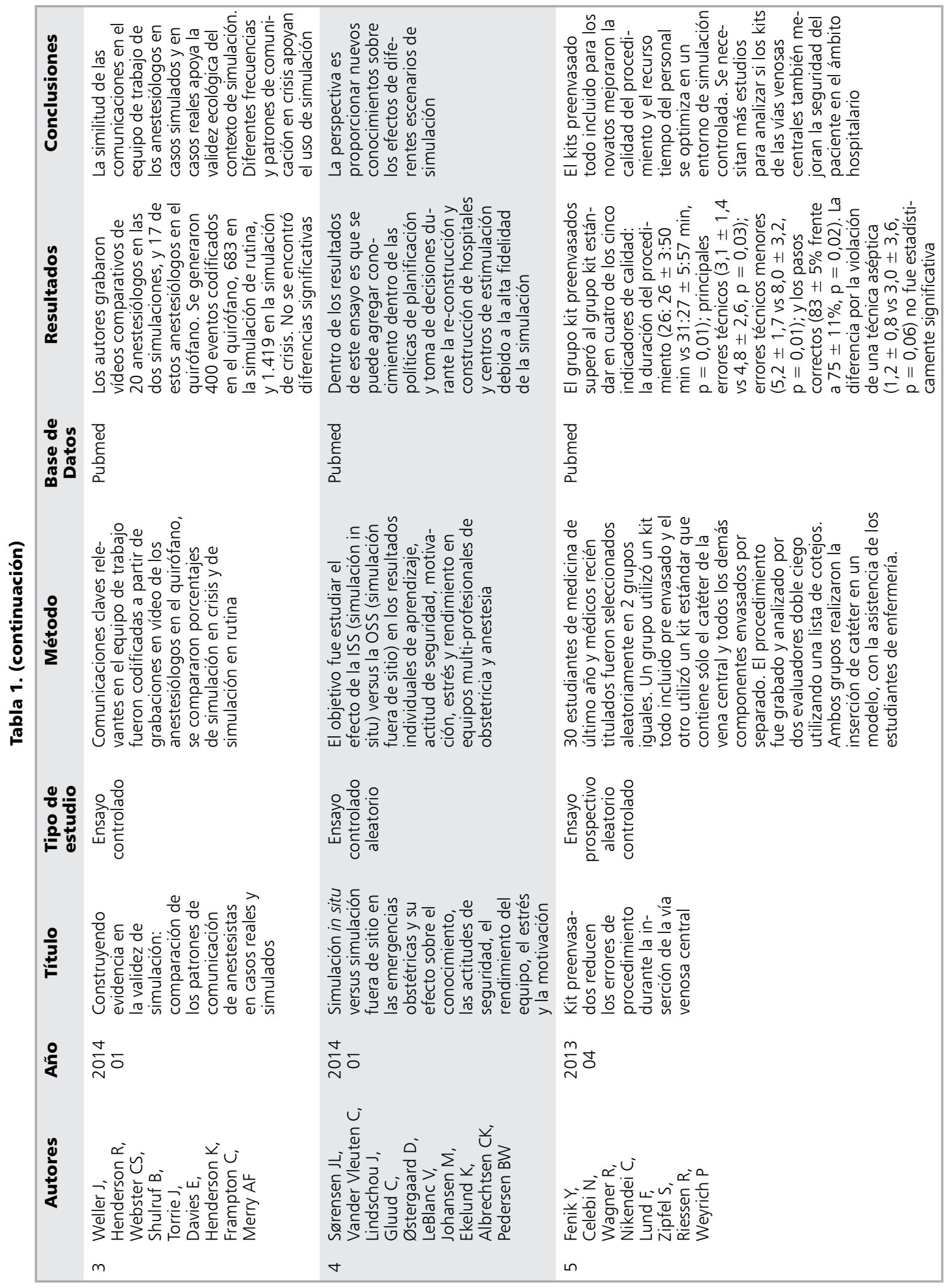




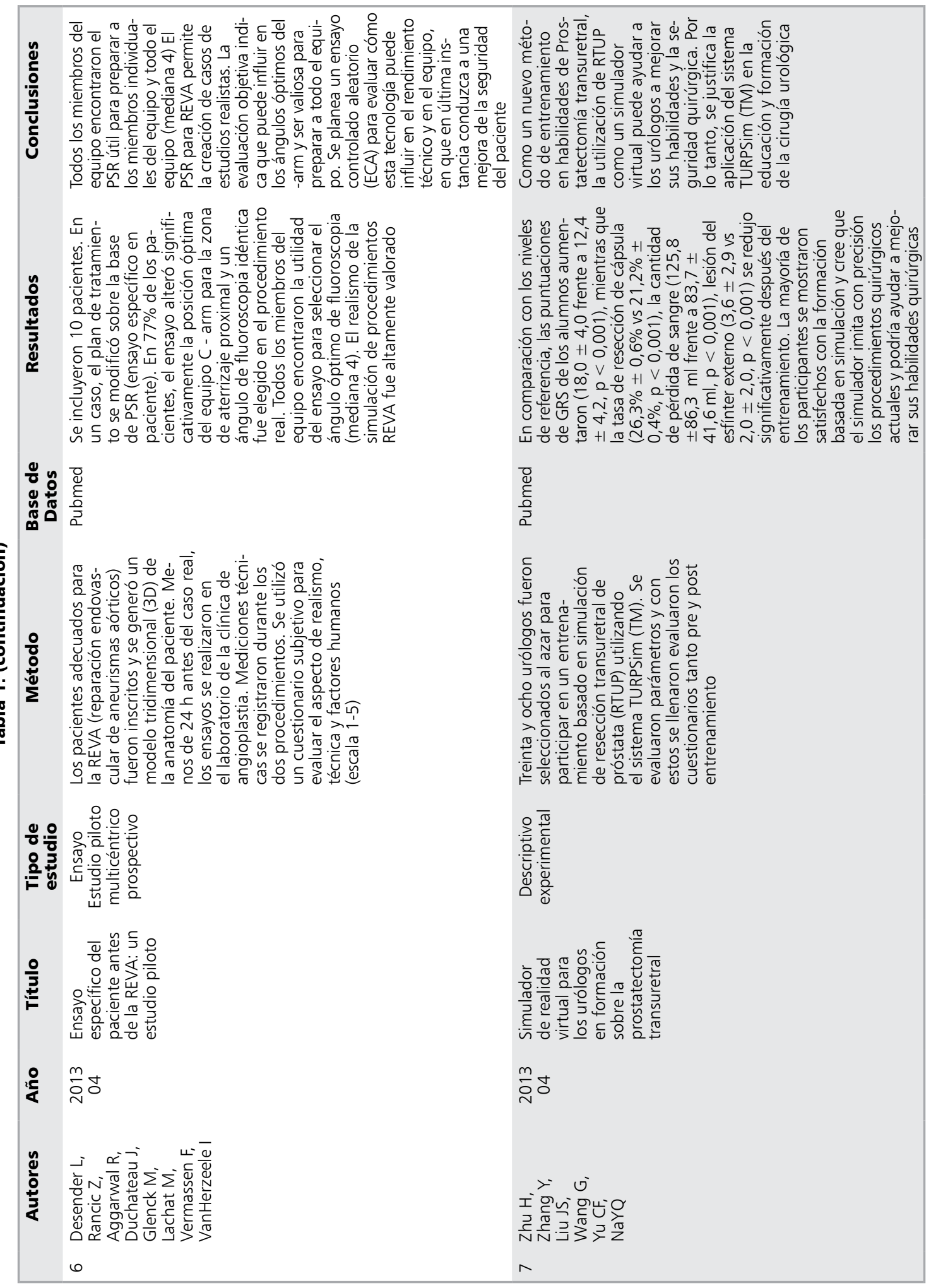




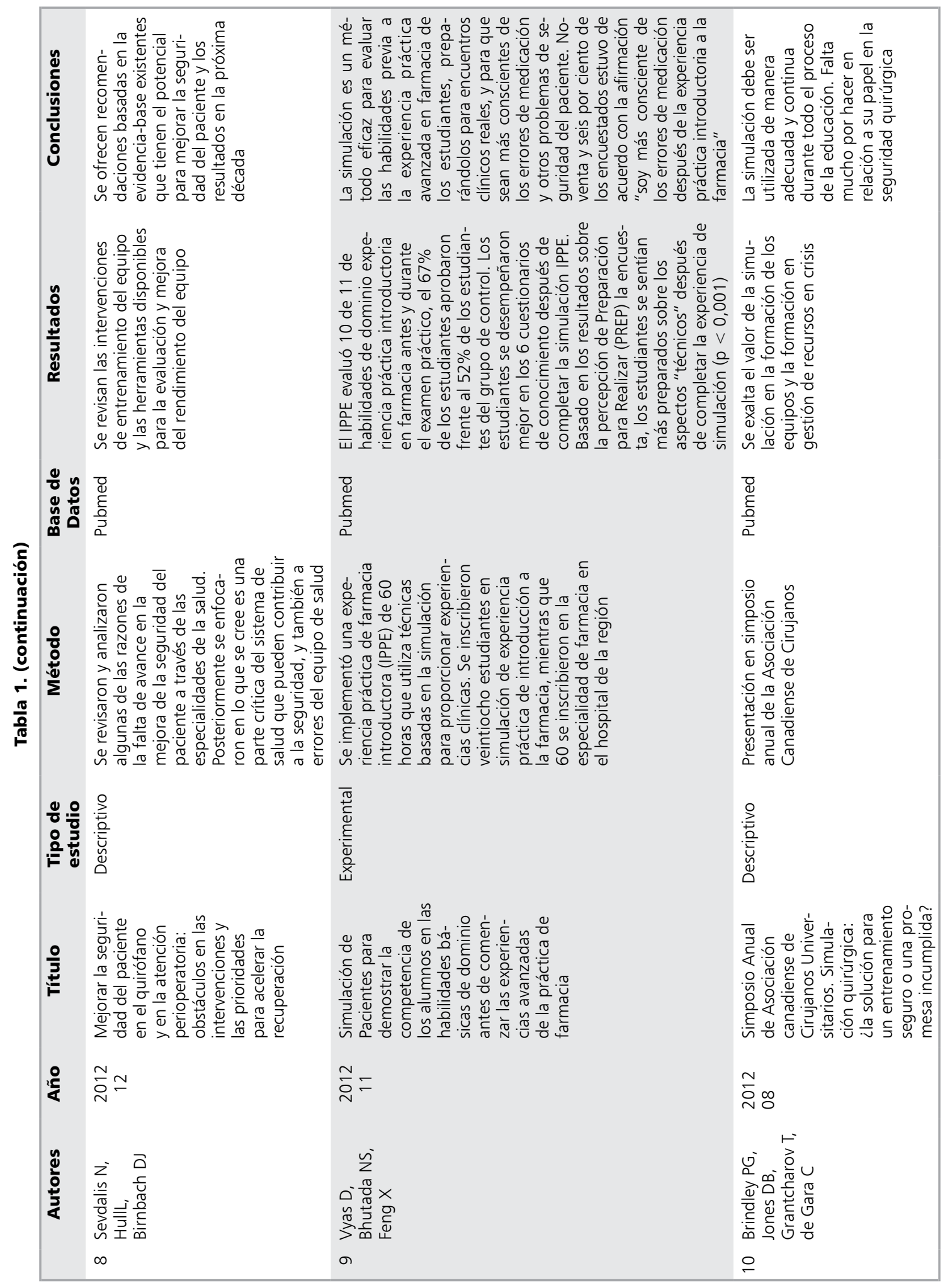




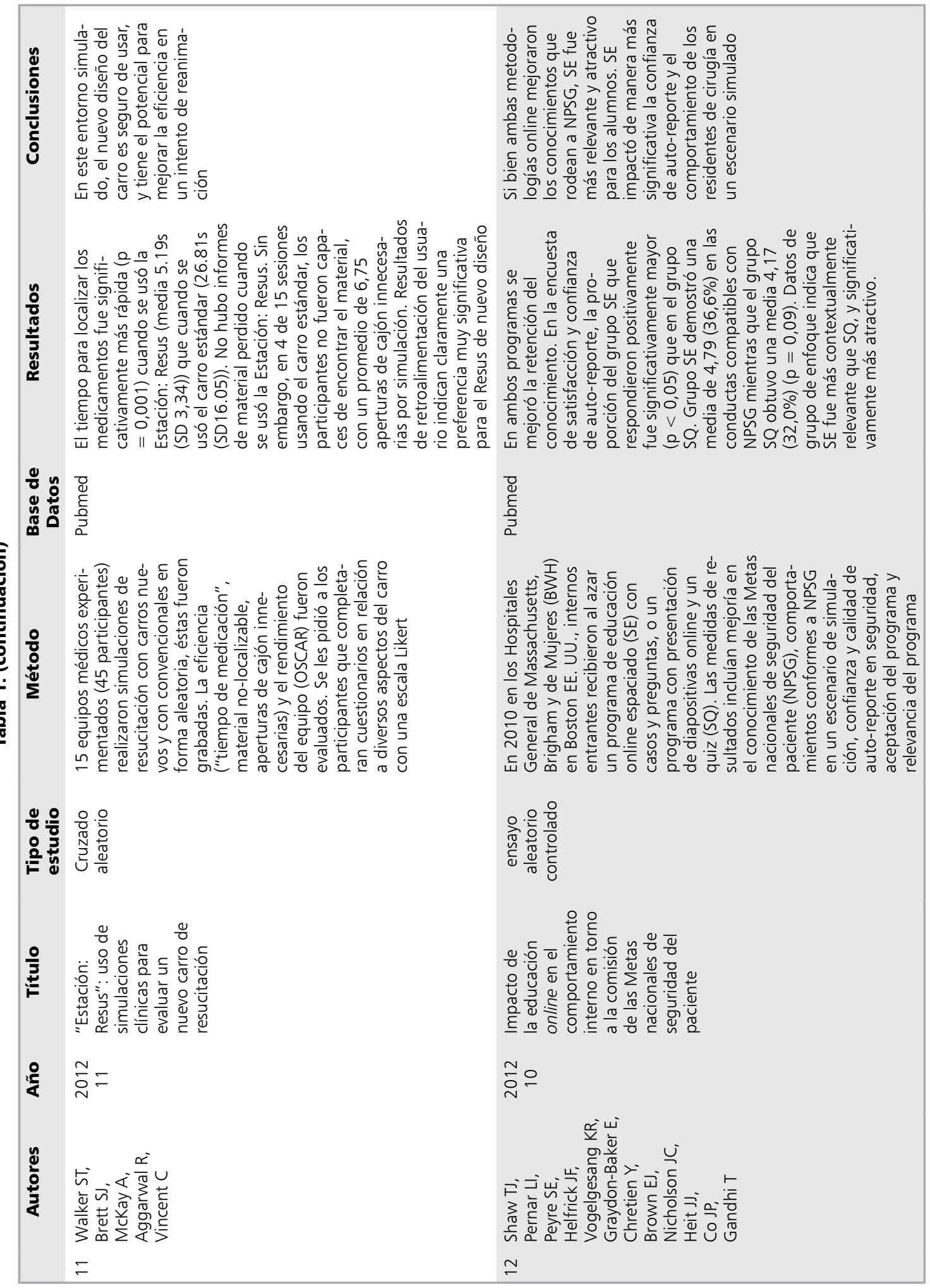




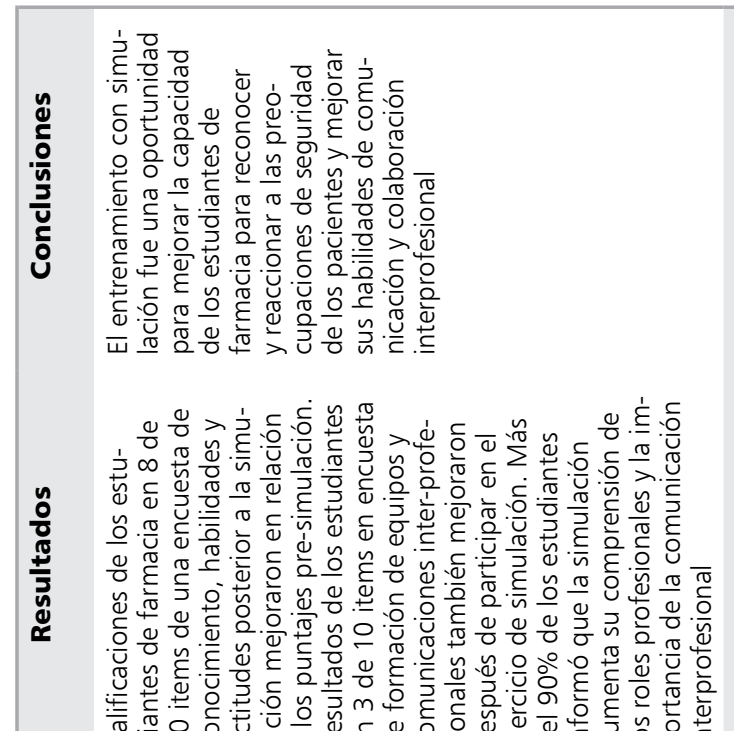

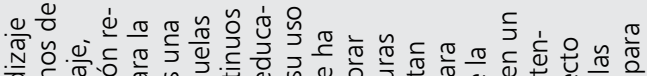

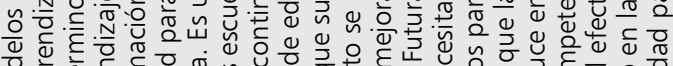

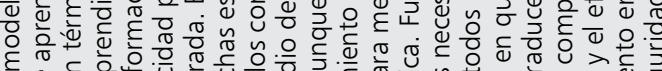

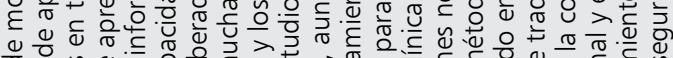

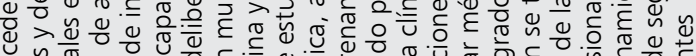
丈

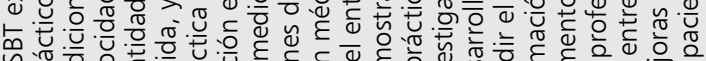

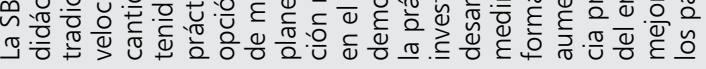

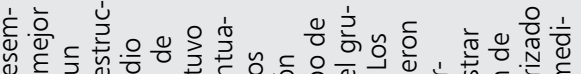

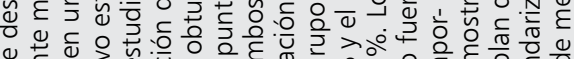

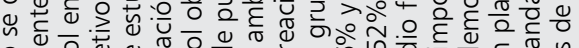

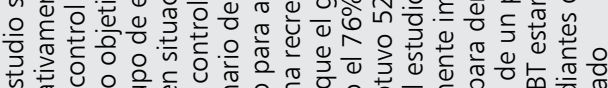

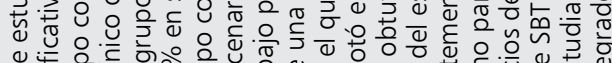

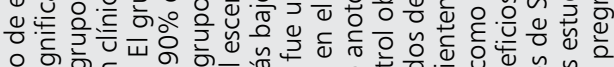

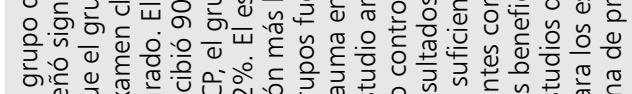

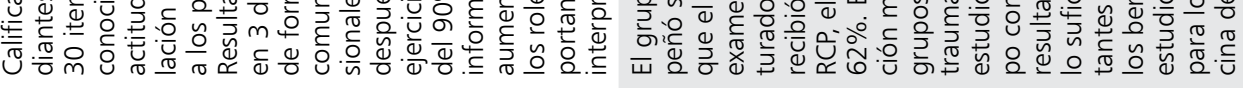

$\stackrel{0}{\varepsilon}$
$\frac{1}{3}$
$\frac{0}{2}$

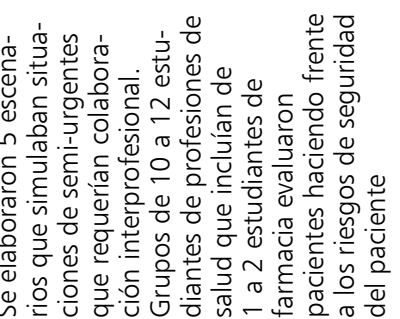

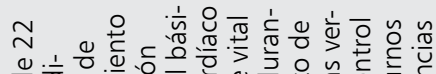

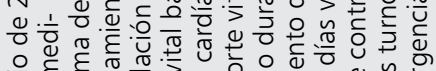

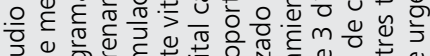

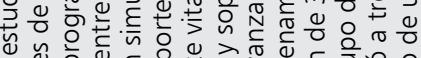

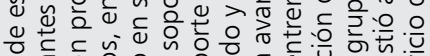

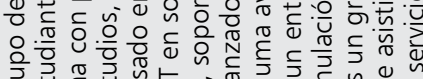

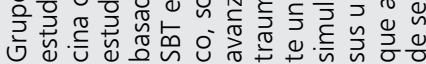

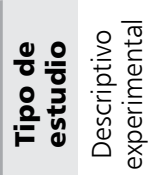

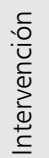
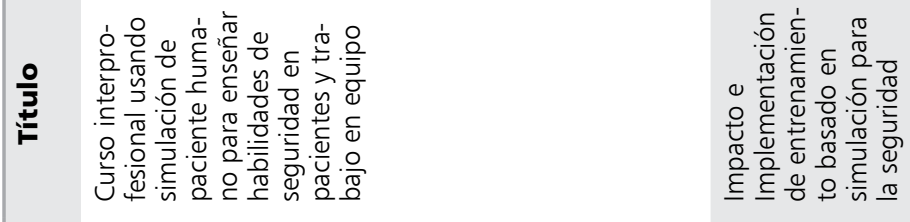

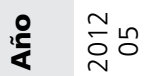

$\stackrel{m}{\frac{m}{N}}$
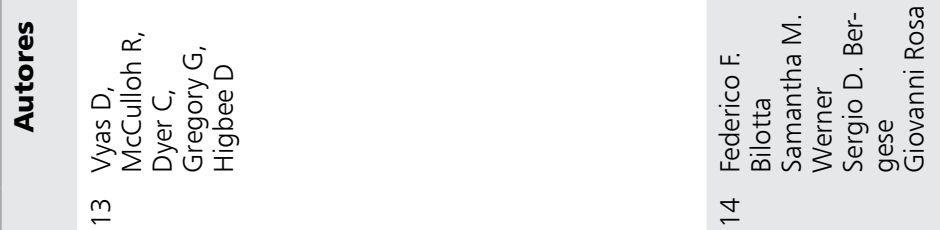


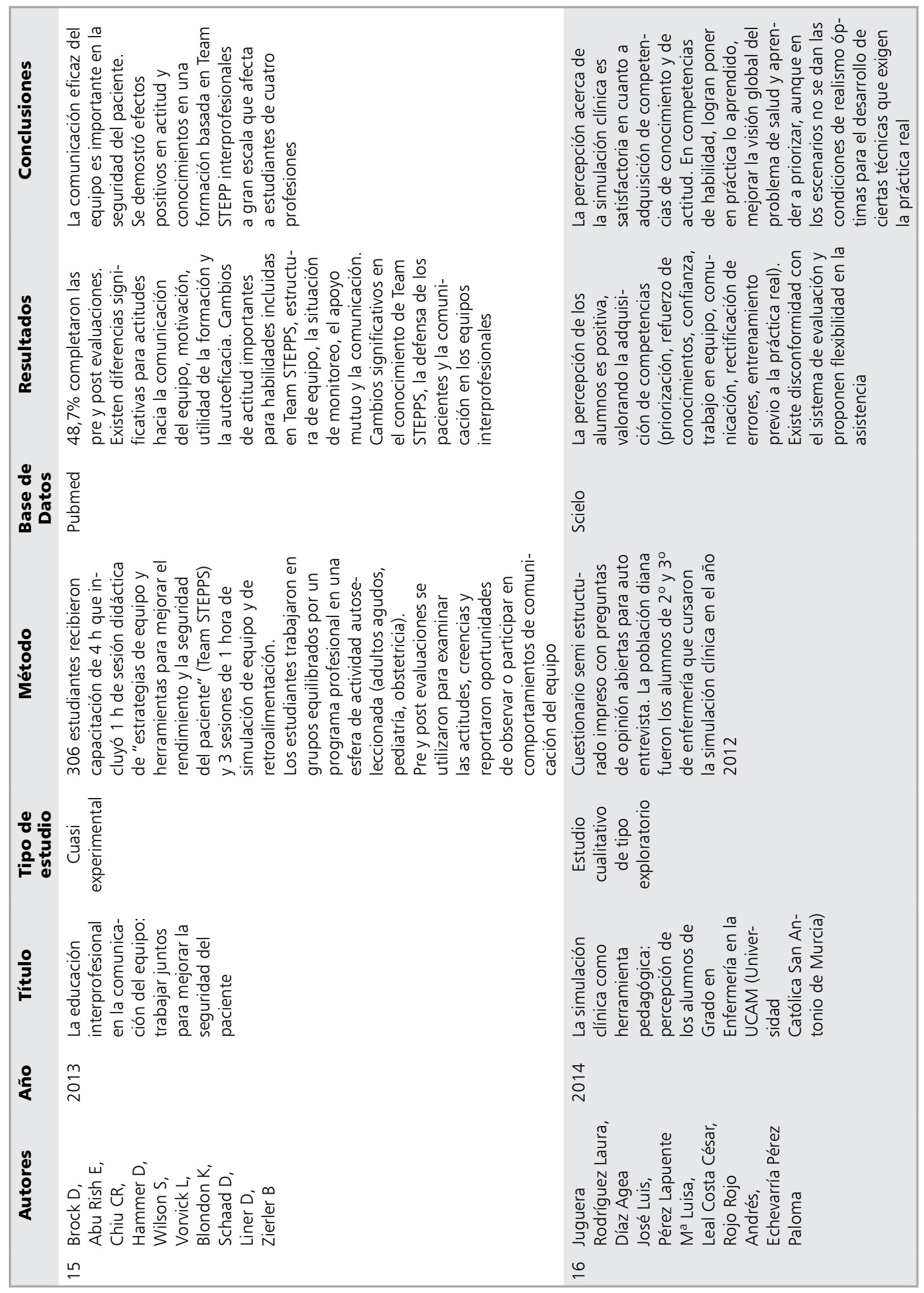




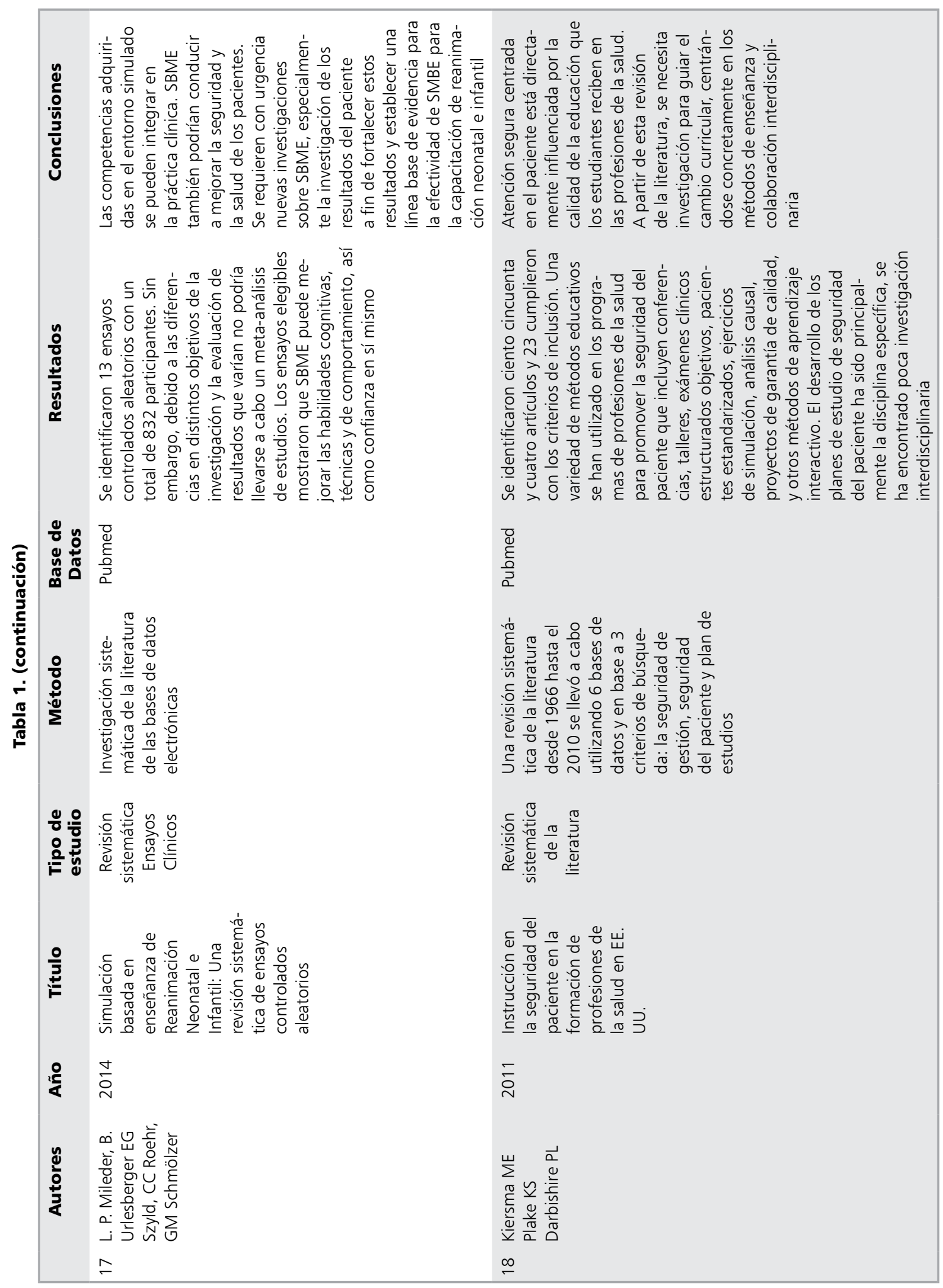




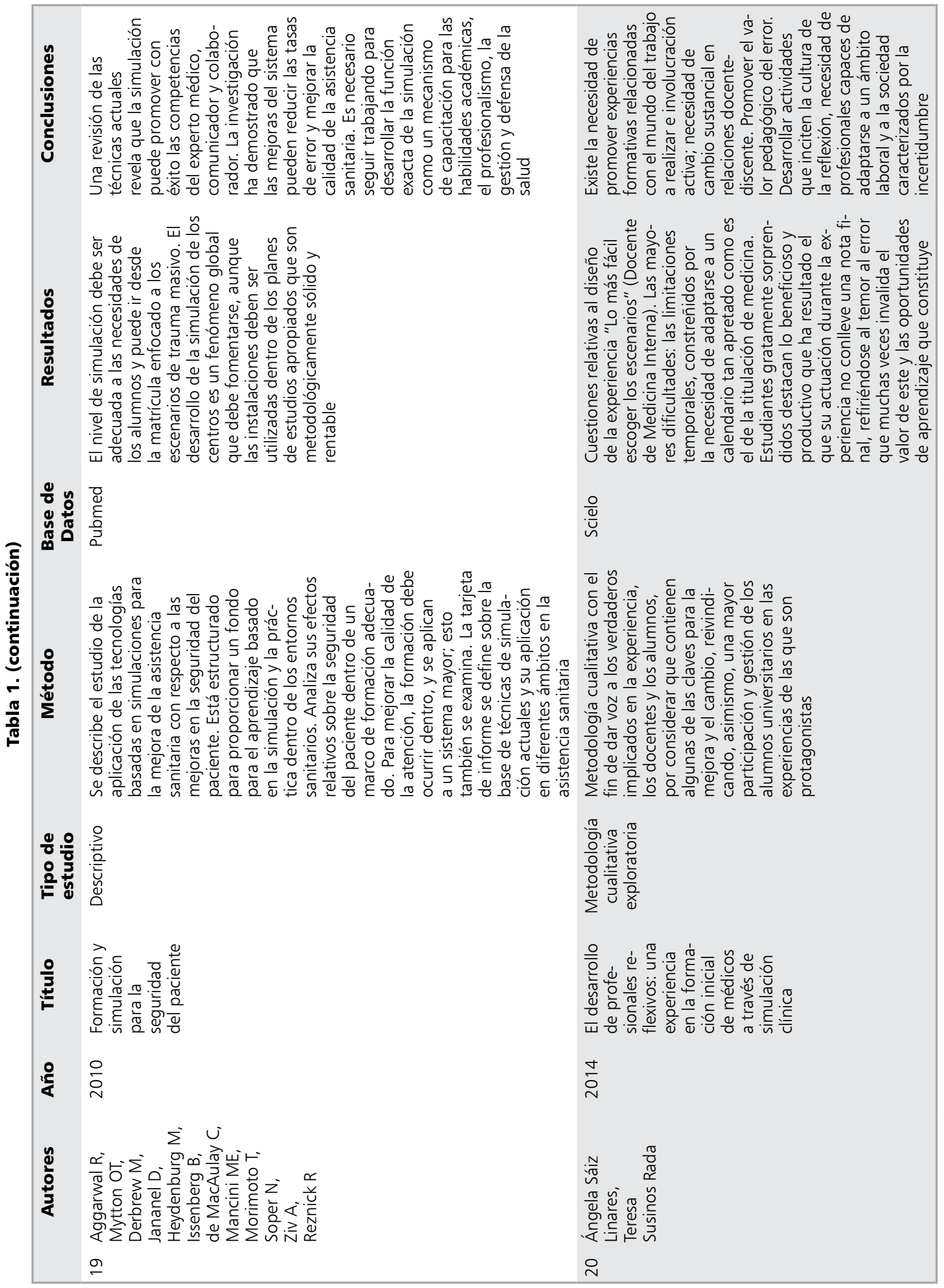




\section{Discusión}

La educación médica se podría ver beneficiada con el uso de metodologías de experiencias simuladas al proporcionar a los estudiantes una visión más realista de la práctica médica. Se necesita evidenciar que esta metodología mejora el logro de las competencias desde la perspectiva de la seguridad del paciente antes del ejercicio de la profesión médica ${ }^{16}$.

Una estrategia propuesta es la inclusión en el currículo de las profesiones de la salud, la formación específica en seguridad del paciente ${ }^{17}$. Los factores que se consideran tienen relación con el liderazgo explícito, la participación temprana de estudiantes de profesiones de la salud; la tutoría realizada por los residentes a los estudiantes acerca de la seguridad del paciente, el uso de la tecnología de información y la promoción del trabajo en equipo entre los profesionales ${ }^{17}$.

Es necesaria la revisión de la literatura para guiar el cambio curricular, centrándose concretamente en los métodos de enseñanza como, por ejemplo, la simulación.

La distribución de los 20 artículos relacionados con el uso de la simulación como metodología educativa está concentrada preferentemente en artículos netamente académicos (30\%) seguido de la especialidad de medicina y cardiología (30\%), anestesiología, cirugía y farmacia (30\%) y finalmente artículos relacionados con obstetricia y urología (10\%).

La seguridad del paciente durante la atención de salud está directamente influenciada por la calidad de la educación que los estudiantes reciben en las profesiones de la salud. El 90\% de los artículos concluye que la simulación contribuye a la seguridad de los pacientes, pues con esta metodología se logra mayor habilidad y destreza en los estudiantes y profesionales egresados de las carreras de la salud.

Los autores de los artículos seleccionados consideran relevantes para la seguridad del paciente los siguientes factores: desarrollo de habilidades de comunicación, colaboración interprofesional $^{7,8}$, gestión en situaciones de emergencias ${ }^{4,9,10} \mathrm{y}$ confianza en los conocimientos adquiridos. Estas competencias se favorecen con el uso de la simulación ${ }^{11}$, aumentando la conciencia del error y su valor pedagógico $^{12,13}$.

En el currículo de la formación del estudiante de farmacia se incorporó la enseñanza de errores de medicación basado en clases con contenido acerca de errores humanos, errores médicos, errores de medicación, proceso de mejora, análisis de causa origen, modo de fallo y análisis de efec$\operatorname{tos}^{18}$. El uso de una metodología activa como es la simulación para encuentros clínicos reales resultó eficaz en los estudiantes de farmacia logrando que, 96\% de los encuestados estuviera de acuerdo con la afirmación "soy más consciente de los errores de medicación después de la simulación"12. Constituye una oportunidad para mejorar la capacidad de los estudiantes de farmacia para reconocer y reaccionar a las preocupaciones sobre seguridad de los pacientes y mejorar sus habilidades de comunicación y colaboración interprofesional ${ }^{7}$. La investigación ha demostrado que las mejoras del sistema pueden reducir las tasas de error y mejorar la calidad de la atención en salud ${ }^{8}$.

En otro estudio ${ }^{13}$ se valora el uso de simulaciones clínicas para mejorar la eficiencia al realizar reanimación con un nuevo carro de paro. Una revisión de las técnicas actuales revela que la simulación puede favorecer el desarrollo de las competencias del experto médico, del comunicador y del colaborador ${ }^{18}$.

De los artículos seleccionados, un autor ${ }^{5}$ declara que la adquisición de habilidades a través de la simulación para mejorar la seguridad del paciente es incierta, encontrando que los resultados no dan lugar a una aceleración de la curva de aprendizaje observando un mayor número de complicaciones en comparación con el grupo control. Se sugiere que un curso estructurado que incluya la formación con el simulador podría tener un impacto negativo en el proceso de aprendizaje en cardio$\operatorname{logí}^{5}$. Otro artículo ${ }^{6}$ afirma que la asociación de la metodología educativa de simulación con la seguridad del paciente se verá en la próxima década.

La literatura refiere como instrumentos de simulación tanto el uso de vídeos o grabaciones como ambientes que representen la clínica ${ }^{11,14}$.

Por lo tanto, la práctica clínica es una actividad que se hace más compleja cada día y está en constante evolución. La combinación de procedimientos, nuevas tecnologías y el factor humano contribuyen a aumentar el riesgo en el proceso asistencial. La atención segura centrada en el paciente está directamente influenciada por la calidad de la educación que los profesionales de la salud reciben, ya que en la medida que mejora 
la curva de aprendizaje mediante el desarrollo de experiencia en ambiente simulado, se minimizarían los riesgos de: a) la práctica en situación real; b) el desempeño del egresado. La revisión de los artículos seleccionados evidencia que una educación basada en la metodología de simulación permite realizar actividades prácticas más seguras para la atención del paciente.

\section{Referencias}

1. Aggarwal R, Mytton OT, Derbrew M, Hananel D, Heydenburg M, et al. Training and simulation for patient safety. Qual Saf Health Care 2010; suppl 2:i 34-43.

2. Baker GR, Norton PG, Flintoft V, Blais R, Brown A, Cox J, Etchells E, et al. The Canadian Adverse Events Study: the incidence of adverse events among hospital patients in Canada. CMAJ 2004; 170 (11): 1678-86.

3. Argullós JL, Palés C, Gomar S. El uso de las simulaciones en educación médica. Teoría de la Educación. Educación y Cultura en la Sociedad de la Información 2010; 11 (2): 147-70.

4. Bilotta FF, Werner SM, Bergese SD, Rosa G. Impact and implementation of simulation-based training for safety. Scientific World Journal 2013; 1-6.

5. Jensen UJ, Jensen J, Olivecrona G, Ahlberg G, Lagerquist $\mathrm{B}$, Tornvall $\mathrm{P}$. The role of a course based on the simulator in coronary angiography performance in the catheterization laboratory of real life. BMC Med Educ 2014; (12) 14: 49.

6. Sevdalis N, Hull L, Birnbach DJ. Improving patient safety in the operating theatre and perioperative care: obstacles, interventions, and priorities for accelerating progress. Br J Anaesth 2012; 109 Suppl 1:i3-i16.

7. Vyas D, Mc Culloh R, Dyer C Gregory G. An Interprofessional couse using human patient simulation to teach patient safety and teamwork skills. Am J, Pharm Educ 2012: 76 (4): 71.

8. Aggarwal R, Mytton OT, Derbrew M, Jananel D, Heydenburg M, Issenberg B, et al. Training and simulation for patient safety Qual Saf Health Care 2010; 19:i34-i43.
9. Walker ST, Brett SJ, McKay A, Aggarwal R, Vincent C. The "Resus: Station": the use of clinical simulations in a randomised crossover study to evaluate a novel resuscitation trolley 2012; 83 (11): 1374-80.

10. Mileder LP, Urlesberger B, Szyld EG, Roehr CC, Schmölzer GM. Simulation-based neonatal and infant resuscitation teaching: a systematic review of randomized controlled trials. Klin Padiatr 2014; 226 (5): 259-67.

11. Weller J, Henderson R, Webster CS, Shulruf B, Torrie J, Davies E, et al. Building the evidence on simulation validity: comparison of anesthesiologists' communication patterns in real and simulated cases. Anesthesiology 2014; 120 (1): 142-8.

12. Vyas D, Bhutada NS, Feng X. Patient simulation to demonstrate students' competency in core domain abilities prior to beginning advanced pharmacy practice experiences. Am J Pharm Educ 2012; 76 (9): 176.

13. Sáiz Linares A, Susinos Rada T. El desarrollo de profesionales reflexivos: una experiencia en la formación inicial de médicos a través de simulación clínica. Revista de Docencia Universitaria 2014; 12 (2).

14. Sørensen JL, Van der Vleuten C, Lindschou J, Gluud C, Østergaard D, LeBlanc V, et al. 'In situ simulation' versus 'off site simulation' in obstetric emergencies and their effect on knowledge, safety attitudes, team performance, stress, and motivation: study protocol for a randomized controlled trial. Trials 2013; 14: 220.

15. Fenik Y, Celebi N, Wagner R, Nikendei C, Lund F, Zipfel S, et al. Prepackaged central line kits reduce procedural mistakes during central line insertion: a randomized controlled prospective trial. BMC Med Educ 2013; 13: 60.

16. Kiersma ME, Plake KS, Darbishire PL. Patient safety instruction in US health professions education. J Pharm Educ 2011; 75 (8): 162.

17. Kirch DG, Boysen PG. Changing the culture in medical education to teach patient safety. Health Aff 2010; 29 (9): 1600-3.

18. Johnson MS, Latif DA, Gordon B. Medication error instruction in schools of pharmacy curricula: a descriptive study. Am J Pharm Educ 2002; 66 (4): 364-71. 